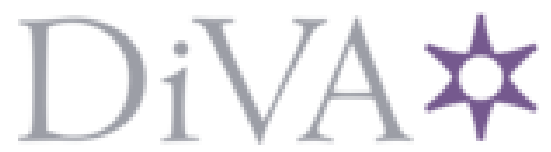

http://www.diva-portal.org

This is the published version of a paper presented at Third International Conference on Applications of Optics and Photonics, Faro, Portugal, May 8-12, 2017.

Citation for the original published paper:

Macedo, A F., Encarnação, T J., Vilarinho, D., Baptista, A M. (2017)

An exploratory study of temporal integration in the peripheral retina of myopes.

In: Proceedings of SPIE 10453 SPIE - International Society for Optical Engineering

Proceedings of SPIE

https://doi.org/10.1117/12.2276237

N.B. When citing this work, cite the original published paper.

Copyright 2017 Society of Photo Optical Instrumentation Engineers. One print or electronic copy may be made for personal use only. Systematic reproduction and distribution, duplication of any material in this paper for a fee or for commercial purposes, or modification of the content of the paper are prohibited.

Permanent link to this version:

http://urn.kb.se/resolve?urn=urn:nbn:se:Inu:diva-67524 


\title{
An exploratory study of temporal integration in the peripheral retina of myopes
}

\author{
Antonio F. Macedo \\ Tito J. Encarnação \\ Daniel Vilarinho \\ António M. G. Baptista
}




\title{
An exploratory study of temporal integration in the peripheral retina of myopes
}

\author{
Antonio F. Macedo (1, a)*, Tito J. Encarnação (b), Daniel Vilarinho (a), António M. G. Baptista (a) \\ (a) Center of Physics, University of Minho, Campus de Gualtar, 4710-057 Braga, Portugal \\ (b) Centro de Diagnóstico da Visão, 5000 Vila Real, Portugal \\ (1*) Linnaeus University, Department of Medicine and Optometry, SE 39182, Kalmar, Sweden. Email: \\ antonio.macedo@lnu.se
}

\begin{abstract}
The visual system takes time to respond to visual stimuli, neurons need to accumulate information over a time span in order to fire. Visual information perceived by the peripheral retina might be impaired by imperfect peripheral optics leading to myopia development. This study explored the effect of eccentricity, moderate myopia and peripheral refraction in temporal visual integration. Myopes and emmetropes showed similar performance at detecting briefly flashed stimuli in different retinal locations. Our results show evidence that moderate myopes have normal visual integration when refractive errors are corrected with contact lens; however, the tendency to increased temporal integration thresholds observed in myopes deserves further investigation.
\end{abstract}

Keywords: Myopia, temporal visual integration, peripheral retina, peripheral refraction

\section{INTRODUCTION}

Myopia is a global problem that affects approximately a billion people worldwide. ${ }^{1,2}$ As a result it is important that we know the mechanisms involved in making an eye myopic and understand the differences in visual perception that occur following the development of the bigger myopic eye. Studies of peripheral refraction have shown that myopes have relative hypermetropic shifts in the periphery while emmetropes have relative myopic shifts. ${ }^{3-5}$ This led to the suggestion that the state of image focus in the peripheral retina might be implicated in refractive error development. ${ }^{6-9}$ These findings have raised the possibility of identifying individuals at risk of developing myopia and eventually the opportunity of preventing its development.

Animal models also suggest that the peripheral retina could have a role in emmetropization and, in particular, in myopia development. An accepted model for emmetropization implies that there is an active feedback mechanism involving the state of focus of the retinal image which drives ocular growth to produce a final refractive state close to emmetropia or, if the habitual viewing distances are relatively short, towards an appropriate level of myopia. ${ }^{7}{ }^{10}$ Smith et al. ${ }^{11}$ damaged the central $10^{\circ}$ of retina around the fovea in infant monkeys, but left the periphery intact: the animals still developed emmetropia. In other studies from the same group, when a hyperopic defocus was imposed in the periphery, but not in the central $10^{\circ}$, animals developed myopia. ${ }^{12}$ Bitzer and Schaeffe $1^{13}$ found that blur caused by a lens can alter biochemical signals in the cells of chicken retina, providing evidence that defocus can alter physiology of the cells. In contrast, the same group of authors failed to show an effect of peripheral refraction in the development of a central refractive error. ${ }^{14}$ Recent longitudinal studies in humans provide evidence that peripheral refraction is correlated with eye elongation and myopia. ${ }^{15-17}$ However, causative effects of the peripheral refraction in myopic development have not been established. Thus, the role of peripheral retina and its optics in myopia development remains under scrutiny.

The temporal processing characteristics of the peripheral retina in myopes have received considerable attention in recent years, in part because there is a lack of understanding of the impact of relative peripheral refractive errors on what the peripheral retina sees. For example, Chen et al. ${ }^{18}$ studied retinal function in humans with myopia using multifocalelectroretinogram (mfERG). These studies showed that high myopes have altered temporal responses. However, participants were evaluated only after their refractive error was as high as 9D. Thus, mfERG results might be showing

Third International Conference on Applications of Optics and Photonics, edited by Manuel F. M. Costa, Proc. of SPIE Vol. 10453, 104532G · @ 2017 SPIE · CCC code: 0277-786X/17/\$18 · doi: 10.1117/12.2276237 
the effect of refractive error mixed with the effect of neural abnormalities. ${ }^{19-21}$ Evidence that myopes have impaired temporal processing has been provided in a recent study by Kuo et al. ${ }^{22}$ Using psychophysics, Kuo found significant differences in interstimulus interval between myopes and emmetropes. These results reinforce the notion that myopes might have temporal visual processing deficits. These deficits may be linked to a mixture of factors such as, neural stretching, neural loss and differences in peripheral refraction.

The aim of the present work was to examine whether temporal integration in the peripheral retina is different between myopes and emmetropes. Recent studies using fMRI showed that lens-induced myopia reduces activity in the visual cortex. ${ }^{23}$ That added to the evidence already given by visual evoked potentials that amplitude in reduced and latency is increased in myopia. ${ }^{24,}{ }^{25}$ Based on previous findings, we would predict that myopes might need longer temporal integration than emmetropes at the periphery of the retina. Only low and moderate myopes were included in the study to avoid the possibilities of advanced neural loss, retinal stretching, visible retinal abnormalities or reduction of acuity associated with higher levels of myopia.

\section{METHODS}

\section{Participants}

We tested the dominant eye of 8 adults (aged 19 to 33 years) with moderate myopia, median $-2.0 \mathrm{D}$ and standard deviation 1.0D (spherical equivalent ranging from -4.25 to $-2.00 \mathrm{D}$, mean cylinder $0.22 \mathrm{D}$ ) and 8 age-matched emmetropes, mean 0.0D and standard deviation $0.30 \mathrm{D}$ (spherical equivalent from -0.63 to $+0.75 \mathrm{D}$, mean cylinder 0.37D). Participants were also matched for on-axis astigmatism (differences were investigated with t-test). All participants had best corrected monocular visual acuity in the tested eye of 10/10 or more, no previous eye disease or anisometropia and no family history of eye disease. On-axis refraction was determined through auto-refraction and refined subjectively. The study conformed to the tenets of the Declaration of Helsinki and was reviewed and approved by the Scientific Committee of the School of Sciences of Minho University. All subjects gave their informed consent to participate.

\section{Apparatus}

Peripheral and central refraction was measured using a photo-refractometer (Power Refractor, Plusoptix, Erlangen, Germany) originally designed for foveal refraction. This device has been considered precise in measuring peripheral refraction for myopia below $6 \mathrm{D}$ and angles up to $40^{\circ} .{ }^{9,26}$

Contrast threshold and integration time were measured using custom programs written in Matlab (MathWorks, Natick, MA) and elements of the Psychophysics toolbox ${ }^{27,28}$ in a PC running windows XP with a NVidea GeForce GTS450 graphics card. The monitor was a Samsung 2233RZ (22", $1680 \times 1050)$, with a refresh rate of $100 \mathrm{~Hz}$ and background luminance of $58 \mathrm{~cd} . \mathrm{m}-2$, this LCD monitor has been extensively tested to be used in psychophysics. ${ }^{29}$

\section{Procedure}

During peripheral refraction measurements the subject sat $1 \mathrm{~m}$ away from the instrument. Fixation targets (black pin button in a white surface) were placed at the same distance and were correctly positioned to measure peripheral refraction at $10^{\circ}, 20^{\circ}$ and $30^{\circ}$, nasally and temporally. Results reported at every angle are the mean of three separate measurements. Fixation targets were held on a flat surface where the central fixation point was in line with the photorefractometer. For measurements off-axis, subjects turned their eye towards the target and the photo-refractometer remained fixed. This set up required a maximum accommodation on-axis of $1 \mathrm{D}$ and a minimum of $0.86 \mathrm{D}$ at $30^{\circ}$, reported refraction values have been corrected for the distance. According to Shen and colleagues soft contact lenses do not have a consistent effect on peripheral relative blur ${ }^{30}$ whilst others showed that axial correction increases the precision of offaxis refractive error measurements. ${ }^{31}$ However, soft contact lens have an effect on relative peripheral refraction (field curvature) that we discuss below. Thus, during measurements participants with myopia were compensated with a spherical contact lens (Focus Dailies ${ }^{\circledR}$, Ciba Vision Corporation) to correct on-axis refractive error, the power of the lens was the spherical equivalent. By correcting central refractive error we also ensured that the required accommodation was similar in both groups. Refractive measurements were converted into power vectors according to the equations below:

$$
\begin{gathered}
\mathrm{M}=\mathrm{S}+\mathrm{C} / 2 ; \\
\mathrm{J} 0=-(\mathrm{C} / 2) \times \cos (2 \alpha) ;
\end{gathered}
$$




$$
\mathrm{J} 45=-(\mathrm{C} / 2) \times \sin (2 \alpha) .
$$

where $\mathrm{M}$ is the spherical equivalent, $\mathrm{S}$ the spherical power, $\mathrm{C}$ the cylindrical power and $\alpha$ the cylindrical axis, J0 (vertical/horizontal astigmatism) and J45 (oblique astigmatism) are the power of the two Jackson cross-cylinder components. ${ }^{32}$

When measuring contrast thresholds, participants practiced before each data collection session by measuring contrast at one randomly-chosen field position: the practice data was discarded. Participants sat $57 \mathrm{~cm}$ away from the screen fixating a black dot at the center. The task in contrast threshold and integration time was to report via button press whether a $6^{\circ}$ vertical Gabor, centered at the position-of-retina, was tilted $30^{\circ}$ to the right or to the left. The perceived size of the Gabor changed slightly with the amount of contrast. ${ }^{33}$ The stimulus was presented to the right or to the left of the fixation point according to the position that we wanted to measure. For $30^{\circ}$ of fixation the dot was shifted from the centre to avoid the stimulus being presented close to the edge of the screen.

Contrast threshold was measured three times in a random order at 6 positions-of-retina: $10^{\circ}, 20^{\circ}$ and $30^{\circ}$ in both the temporal and the nasal retina. Threshold was calculated using Quest, an adaptive psychometric method included in the psychophysics toolbox. ${ }^{34}$ For testing at $10^{\circ}$ and $20^{\circ}$ the spatial frequency was set at 4 cycles-per-degree and at $30^{\circ}$ spatial frequency was set at 2 cycles-per-degree. These values were calculated to be approximately twice the resolution threshold, for the position-of-retina tested, according to Rovamo et al ${ }^{35}$ As shown in Figure 1-A, the target was preceded by a 50 milliseconds (ms) duration cue, exposed for $200 \mathrm{~ms}$, and followed by a blank screen until a response was given.

Contrast threshold was measured to determine the contrast of the Gabor to be used when measuring the integration time for each participant at each position of the retina. The use of individual contrast thresholds ensured that the task of visual integration was equally difficult for all participants.

Temporal integration was measured twice in random order in the same 6 positions and conditions described for contrast threshold. Contrast of the Gabor for each subject was set at twice their contrast threshold. In each block, integration time was determined using the method of constant stimuli based on 400 trials per retinal location ( 80 trials per exposure). As shown in Figure 1-B, the target was preceded by a 50 ms duration cue, exposed for variable periods of 10, 30, 60, 90 and $140 \mathrm{~ms}$, selected in random order, and followed by a noise mask until a response was given.
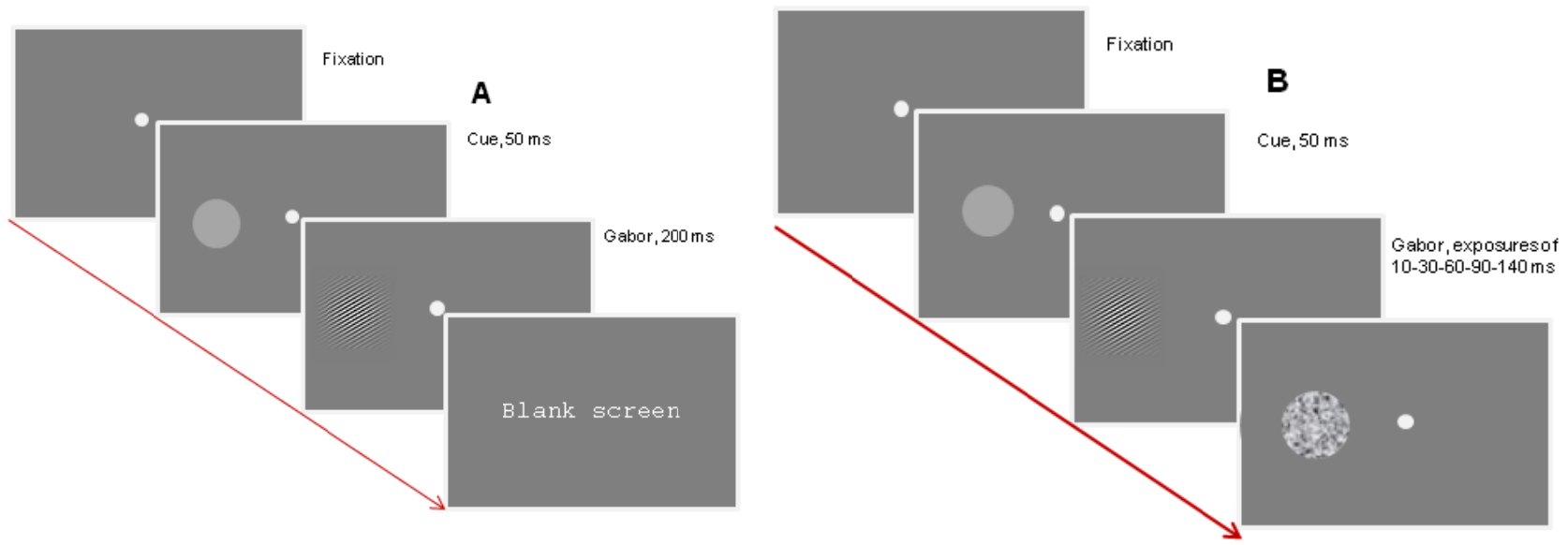

Figure 1: Stimuli sequence for (A) contrast threshold measurements and (B) temporal integration measurements. In both sequences a beep was presented simultaneously with the target (Gabor).

The integration threshold was computed by fitting a sigmoid function in Origin (V8.5.0, OriginLab Corp.,Northampton, MA, USA) and defined as the exposure time yielding $75 \%$ of correct responses as shown in Figure 2. 

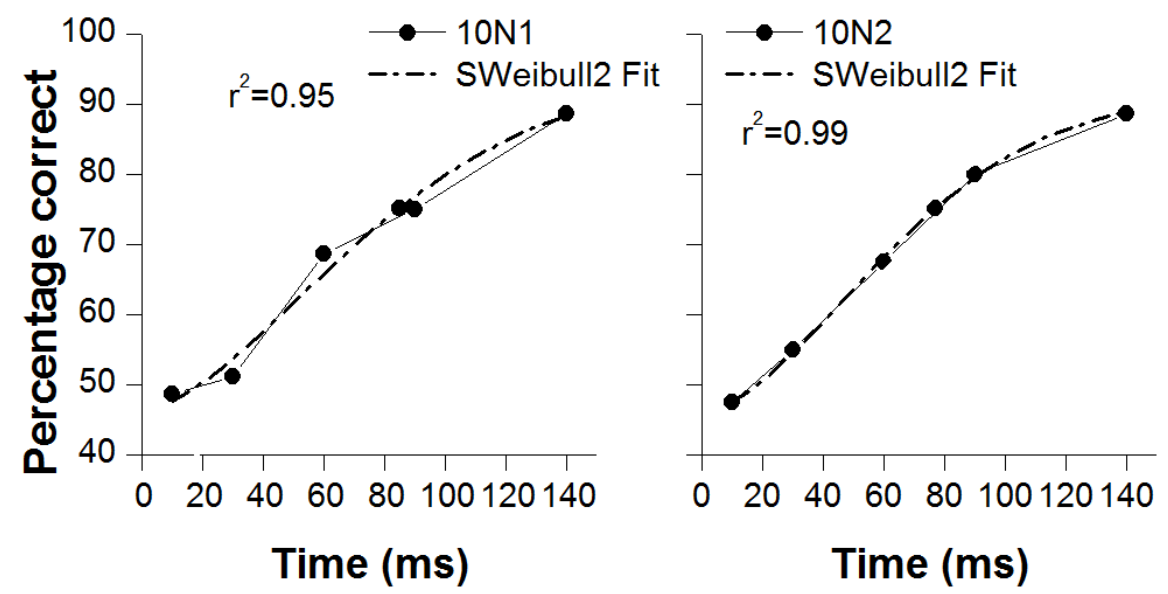

Figure 2: Example of two fitted functions to obtain temporal integration thresholds in two different blocks (10N1 and 10N2) of the same participant at 10 degrees, nasal retina.

\section{Data analysis}

In order to test for differences between myopes and emmetropes in our repeated measures design we performed a linear mixed model analysis for each dependent variable. ${ }^{36}$ In these models fixed factors were "group" and "position-of-retina". Bonferroni correction was applied for multiple comparisons. "Subjects" was assigned as a random factor, the analysis was conducted in SPSS (IBM Corporation, v21). Correlations were tested to assess associations between outcome measures.

\section{RESULTS}

\section{Psychophysical results}

Threshold for integration are shown in Figure 3 and summarized in Table 1. The estimated marginal means (EMM) combining all positions measured was $69.8 \mathrm{~ms}$ (standard error $=5.8 \mathrm{~ms}$ ) for myopes and $64.0 \mathrm{~ms}$ (standard error $=5.9$ $\mathrm{ms})$ for emmetropes. The effect of position-of-retina was statistically significant, $F(5,144)=4.19, \mathrm{p}<0.001$. The effect of group was not statistically significant $(\mathrm{p}=0.50)$. The interaction group $\times$ position-of-retina was also not statistically significant $(\mathrm{p}=0.92)$.

Comparing the EMM for each position, the mean difference between $30 \mathrm{~T}$ and $30 \mathrm{~N}$ was $20 \mathrm{~ms}(\mathrm{p}=0.01)$, the mean difference between $20 \mathrm{~T}$ and $10 \mathrm{~N}$ was $18 \mathrm{~ms}(\mathrm{p}=0.037)$, the mean difference between $20 \mathrm{~T}$ and $20 \mathrm{~N}$ was $18 \mathrm{~ms}(\mathrm{p}=0.043)$ and the mean difference between $20 \mathrm{~T}$ and $30 \mathrm{~N}$ was $26 \mathrm{~ms}(\mathrm{p}=0.001)$. These results show a significant difference in temporal integration between positions at the nasal and at the temporal retina. Detailed results separated by group and position-of-retina are summarized in Table 1.

The effect of group in contrast sensitivity was not statistically significant $(\mathrm{p}=0.77)$, the effect of position-of-retina was statistically significant, $\mathrm{F}(5,262)=125.3, \mathrm{p}<0.001$. The interaction group $\times$ position-of-retina was not significant $(\mathrm{p}=0.85)$, results are summarized in Table 1 . 


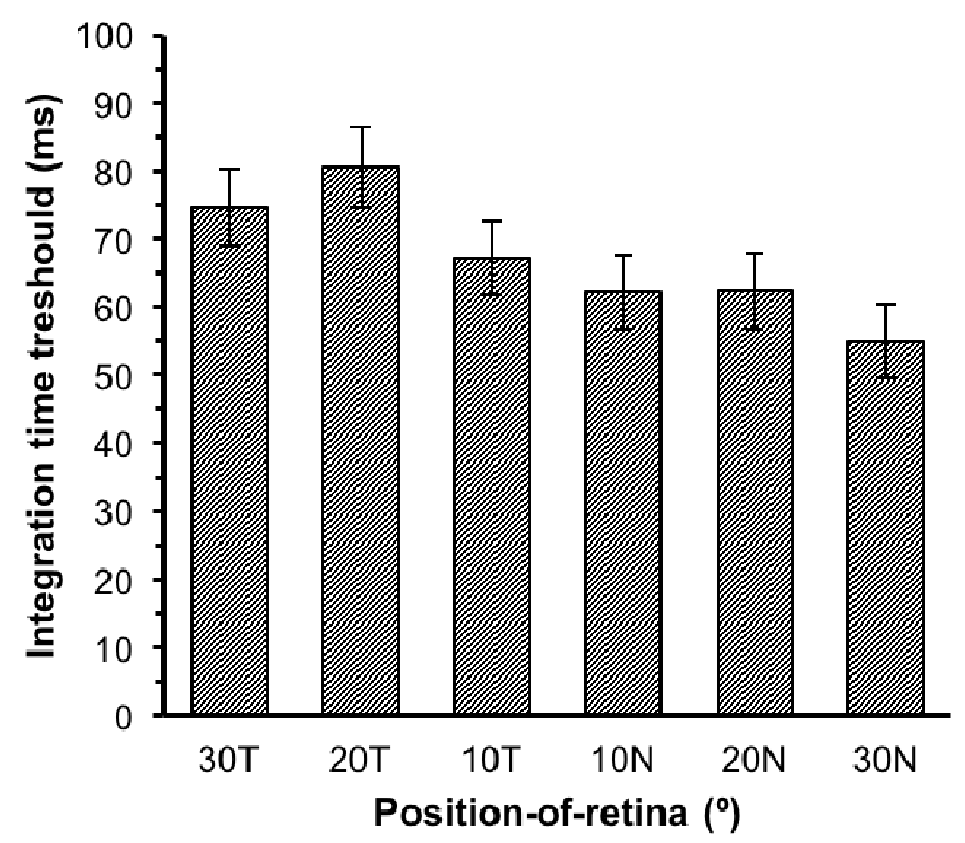

Figure 3: Integration time for the 6 position-of-retina. On the $\mathrm{x}$ axis, T letters indicate positions in the temporal retina and $\mathrm{N}$ letters indicate positions in the nasal retina. Differences between $30 \mathrm{~T}$ and $30 \mathrm{~N}, 20 \mathrm{~T}$ and $10 \mathrm{~N}, 20 \mathrm{~T}$ and $20 \mathrm{~N}$ and between $20 \mathrm{~T}$ and $30 \mathrm{~N}$ were statistically significant. The mean values shown are the EMM resulting from the linear mixed models analysis, error bars show the standard error for the EMM.

Table 1: Results for temporal integration and contrast sensitivity separated by position-of-retina and group. In the position-of-retina column numbers indicate eccentricity in degrees, $T$ letters indicate positions in the temporal retina and $\mathrm{N}$ letters indicate positions in the nasal retina. The mean values are the estimated marginal means (EMM) resulting from the linear mixed models analysis, SE are the standard errors for the EMMs.

\begin{tabular}{|c|c|c|c|c|c|}
\hline \multirow[t]{2}{*}{ group } & \multirow[t]{2}{*}{ position-of-retina } & \multicolumn{2}{|c|}{$\begin{array}{c}\text { temporal integration } \\
(\mathrm{ms})\end{array}$} & \multicolumn{2}{|c|}{ Log10(contrast sensitivity) } \\
\hline & & mean & SE & mean & SE \\
\hline \multirow{6}{*}{ emmetropes } & $30 \mathrm{~T}$ & 68.7 & 8.0 & 1.4 & 0.074 \\
\hline & $20 \mathrm{~T}$ & 80.7 & 8.7 & 1.3 & 0.074 \\
\hline & $10 \mathrm{~T}$ & 62.9 & 7.7 & 2.1 & 0.074 \\
\hline & $10 \mathrm{~N}$ & 58.2 & 7.5 & 2.1 & 0.074 \\
\hline & $20 \mathrm{~N}$ & 60.5 & 7.8 & 1.8 & 0.074 \\
\hline & $30 \mathrm{~N}$ & 53.5 & 8.0 & 2.1 & 0.074 \\
\hline \multirow{6}{*}{ myopes } & $30 \mathrm{~T}$ & 80.5 & 7.8 & 1.4 & 0.074 \\
\hline & $20 \mathrm{~T}$ & 80.1 & 7.8 & 1.2 & 0.074 \\
\hline & $10 \mathrm{~T}$ & 71.2 & 7.5 & 2.2 & 0.074 \\
\hline & $10 \mathrm{~N}$ & 66.2 & 7.8 & 2.0 & 0.074 \\
\hline & $20 \mathrm{~N}$ & 64.2 & 7.7 & 1.8 & 0.074 \\
\hline & $30 \mathrm{~N}$ & 56.5 & 7.5 & 2.1 & 0.074 \\
\hline
\end{tabular}




\section{Refractive results}

Results computed directly from the photo-refractometer readings are defined as raw $M$ values. A model with fixed factors "group" and "position-of-retina", random factor "subjects" was constructed to test the effect of these factors in raw $M$. As would be expected, the myopic group showed an overall more negative $M, F(5,258)=237$, $p<0.001$, mean difference between groups was $2.25 \mathrm{D}$. The effect of position-of-retina or interaction position-of-retina $\times$ group was not statistically significant $(\mathrm{p}=0.21)$. We ran the same model but now $\mathrm{M}$ was normalized by subtracting the central raw $\mathrm{M}$ from the raw $\mathrm{M}$ of each position-of-retina, results are shown in Figure 4. The effect of group $(\mathrm{p}=0.82)$, position-of-retina $(\mathrm{p}=0.60)$ and the interaction group $\times$ position-of-retina $(\mathrm{p}=0.86)$ were not statistically significant.

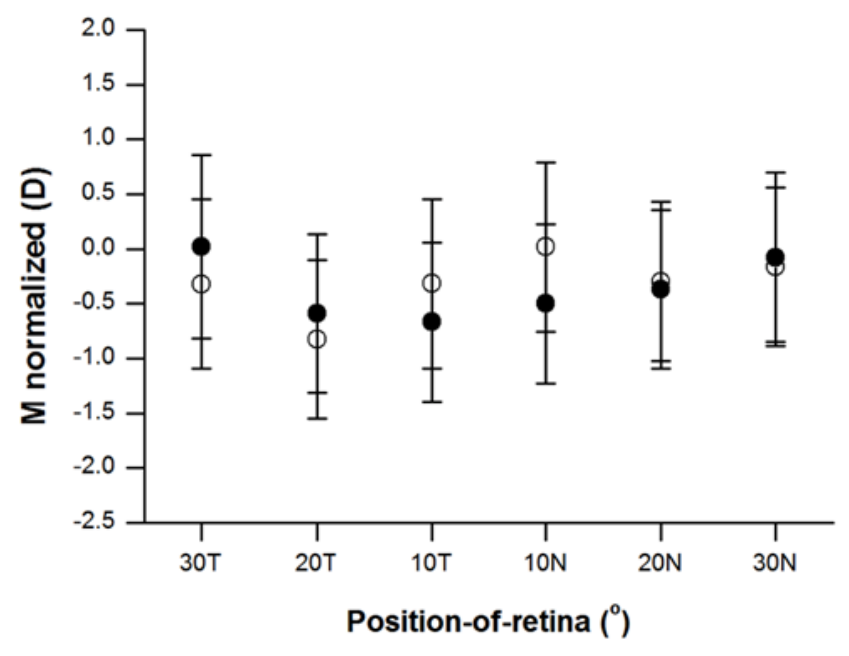

Figure 4: Variation of normalized $\mathrm{M}$ with eccentricity for emmetropes (open symbols) and myopes (filled symbols). On the $\mathrm{x}$ axis, $\mathrm{T}$ letters indicate positions in the temporal retina and $\mathrm{N}$ letters indicate positions in the nasal retina. Error bars show the $95 \%$ confidence interval.

Raw J0 values are defined as results computed directly from the photo-refractometer readings. Here we report a statistical analysis of normalized J0 only because raw and normalized produced similar results in our model. Normalization was performed with $\mathrm{J} 0$ as described above for M value. A model with fixed factors "group" and "position-of-retina", random factor "subjects" was run, results are shown in Figure 5 (see also supplementary Table 1 in supplementary results). The effect of position-of-retina was statistically significant, $F(5,276)=37.5, p<0.001$. The effect of group $(\mathrm{p}=0.29)$ and the interaction position-of-retina $\times$ group $(\mathrm{p}=0.89)$ were both not statistically significant. These results show that astigmatism increases with eccentricity at a higher rate in the temporal retina in both groups. ${ }^{37}$

We performed for J45 the same type of normalization and statistical analysis that we performed for J0. Results are shown in Figure 6. The effect of position-of-retina was statistically significant, $F(5,276)=23.2, p<0.001$. The effect of group $(\mathrm{p}=0.430)$ and the interaction position-of-retina $\times$ group $(\mathrm{p}=0.203)$ were both not significant. Pairwise comparisons indicate that J45 in the peripheral retina was small but significantly more negative in positions of the nasal retina than in the temporal in both groups (see also supplementary Table 2 in supplementary results). These results are in line with what has been found by other authors. ${ }^{38}$ 


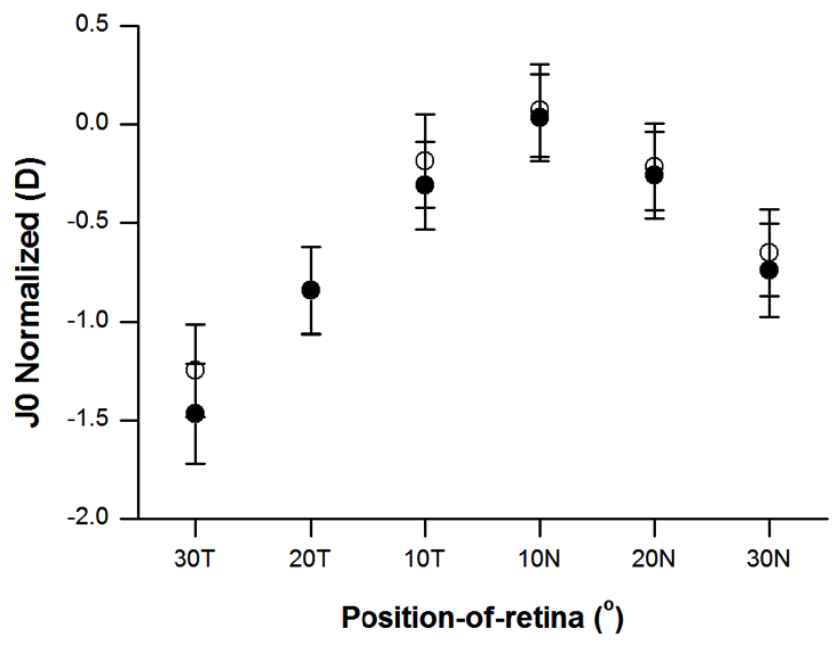

Figure 5: Variation of $\mathrm{J} 0$ with eccentricity for emmetropes (open symbols) and myopes (filled symbols). On the $\mathrm{x}$ axis, $\mathrm{T}$ letters indicate positions in the temporal retina and $\mathrm{N}$ letters indicate positions in the nasal retina. Error bars show the $95 \%$ confidence interval.

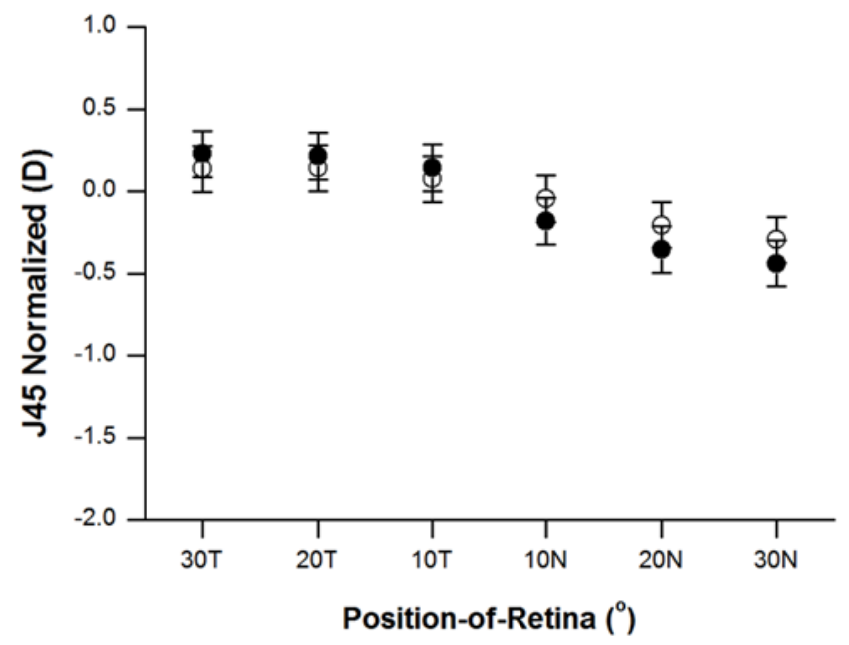

Figure 6: Variation of J45 with eccentricity for emmetropes (open symbols) and myopes (filled symbols). On the $\mathrm{x}$ axis, T letters indicate positions in the temporal retina and $\mathrm{N}$ letters indicate positions in the nasal retina. Error bars show the $95 \%$ confidence interval.

\section{Correlation between outcome measures}

We tested for correlation between refractive results (M, J0, J45), contrast sensitivity and temporal integration per each position-of-retina. A negative correlation was found between contrast sensitivity and temporal integration at 10 degrees nasal, $r=-0.54(\mathrm{p}<0.031)$. These results indicate that, even when stimulus contrast was optimized by subject and position-of-retina, in this position participants better contrast sensitivity led to lower temporal integration thresholds. 


\section{DISCUSSION}

In this study we investigated temporal integration thresholds in myopes and emmetropes. We hypothesized that myopes would have different (extended) temporal integration times. However, we failed to find significant differences between myopes and emmetropes for temporal integration in the peripheral retina. We found differences in visual integration amongst positions of the retina and differences in the amount of astigmatism, represented by J0 and J45 in our results. The temporal retina tends to have prolonged integration times when compared with nasal retina and the amount of vertical/horizontal astigmatism was also higher in the temporal retina.

Temporal integration in myopes was not different from emmetropes. We intentionally included only moderate myopes because these are less likely to suffer from other abnormalities of the retina such as stretching and neural loss that people with high myopia can suffer. ${ }^{19,21}$ Our results for integration time are in agreement with other studies. For example, Hess and Maehara ${ }^{39}$ measured the delay that the visual system takes to consciously perceive brief temporal events. They found that it takes $67 \mathrm{~ms}$ in the fovea and $91 \mathrm{~ms}$ at $5^{\circ}$ to perceive brief presented visual stimulus. Our results are also in line with other studies showing equal performance of myopes $(>6 \mathrm{D})$ and emmetropes, viewing centrally, in tasks such as spatial and dynamic spatial contrast sensitivity and temporal modulation sensitivity. ${ }^{40}$ Conversely, Kou et al. (2012) studied visual processing using visual backward masking and found differences between myopes and emmetropes. ${ }^{22}$ These results are not in conflict with our study because we were testing a distinct area with a different stimulus. Kuo tested letters parafovealy and we tested Gabors at greater eccentric locations. In addition, our participants were less myopic.

We found a difference in temporal integration thresholds between positions of the temporal and nasal retina. The nasaltemporal asymmetry is hard to understand based on the expected structural changes in myopes versus emmetropes. Different studies found that eye expansion (cornea-to-retina length) with increasing myopia is more pronounced in the nasal side. ${ }^{41,}{ }^{42}$ As Logan and colleagues observed that this finding counteracts what would be expected based on environmental factors and it remains without explanation. ${ }^{42}$ In contrast, superior performance of the nasal retina has been found in functional studies using electrophysiology ${ }^{43}$ and psychophysics. ${ }^{44-46}$ It is known that the nasal retina has more ganglion cells than the temporal retina ${ }^{47}$ and that is the most likely reason for our findings. However, because we used Gabors of different spatial frequencies and contrasts we need to consider our differences between retinal positions carefully. In brief, our results for temporal integration confirmed what was expected for normal observers but failed to show differences between myopes and emmetropes.

The variation of refractive error with position of the retina was similar in both groups. Our results for spherical equivalent or $\mathrm{M}$ at peripheral positions of the retina are in line with previous research studying peripheral refraction in children with low myopia ${ }^{16}$ or young adults. ${ }^{48}$ Sng et al. (2011) found relative hyperopia in myopes only in one position of the retina - $30^{\circ}$ temporal - and no differences at $15^{\circ}$ temporal or in equivalent positions in the nasal retina. ${ }^{16}$ In this and other studies myopes with moderate and high myopia showed relative hyperopia in all peripheral positions. ${ }^{3,16}$ Our peripheral refraction measurements were performed with soft contact lens and that can cut the degree of hyperopic field curvature as shown by different authors. ${ }^{30,49}$ The method we used to measure peripheral refraction might also raise the question whether our relative peripheral refractive error has been affected by accommodation. However, Mathur et al. ${ }^{50}$ have shown that these values would not be significantly altered by using a measuring technique that requires some accommodation. Astigmatism variation, J0 and J45 with position of the retina was also similar for myopes and emmetropes. Other studies also showed that astigmatism is independent of the central refractive error. ${ }^{16,48,51}$ We found higher astigmatism in the peripheral retina, which is in line with the findings of others studies summarized by Charman. ${ }^{7}$

Refractive power was not correlated with visual performance. Spherical error was small, similar in different positions of the retina and not correlated with visual performance. That is in agreement with previous findings showing no effect of small spherical errors (close to 2D), for example, in resolution acuity in the periphery. According to Anderson (1996), this happens because the peripheral retina is sampling limited. ${ }^{52}$ There was no correlation but a coincidence that $\mathrm{J} 0$ and temporal integration were both higher in the temporal retina than in the nasal retina. These results seem to indicate that astigmatism can impair visual performance. This is in agreement with a recent study by Lewis et al. ${ }^{53}$ showing that peripheral acuity for low contrast letters can be improved when astigmatism higher than 1D is corrected. It is known that astigmatism can cause strong form deprivation. ${ }^{37,54,55}$ It is also known that astigmatism can have a pronounced detrimental effect on critical fusion frequency for small light sources. ${ }^{56}$ Thus, we speculate that astigmatism is contributing to the poorer visual performance of the temporal retina. 
This study present two main limitations: 1) the accuracy of our experimental setup to measure contrast threshold and 2) limited statistical power due small sample $(\mathrm{n}=16)$. The reduced accuracy of contrast measurements was mainly due to the range of 8 bits in our graphics card. However, because contrast was just an intermediate measure, we believe that the main outcome of this study, temporal integration thresholds, was not affected by this. To check whether our nonsignificant differences in temporal integration between groups were due to a lack of statistical power we conducted post hoc power analyses. We used GPower with power $(1-\beta)$ set at $0.80, \alpha=005 .{ }^{57}$ The analysis revealed that for the between-groups comparison effect size observed in the present study $(\mathrm{d}=0.18)$, an $\mathrm{n}$ of approximately 35 would be needed to obtain statistical power at the recommended 0.80 level.

In conclusion, moderate myopes do not show differences in temporal integration in the peripheral retina when compared with emmetropes. Further studies, with more participants and myopes with higher refractive error are desirable to explore whether myopes with relative hyperopia in the periphery show temporal integration deficits. 


\section{ACKNOWLEDGMENTS}

Parts of these results have been presented at ARVO 2012 annual meeting. We would like to acknowledge Associação de Profissionais Licenciados em Optometria (APLO) for their financial support and Inês Sousa PhD for her help with the statistics.

\section{REFERENCES}

[1] T. T. Norton, R. Manny, and D. J. O'leary, "Myopia—global problem, global research," Optometry \& Vision Science, 82(4), 223-225 (2005).

[2] J. H. Kempen, P. Mitchell, K. E. Lee et al., "The prevalence of refractive errors among adults in the United States, Western Europe, and Australia," Arch Ophthalmol, 122(4), 495-505 (2004).

[3] D. A. Atchison, N. Pritchard, and K. L. Schmid, "Peripheral refraction along the horizontal and vertical visual fields in myopia," Vision Res, 46(8-9), 1450-8 (2006).

[4] D. A. Atchison, N. Pritchard, K. L. Schmid et al., "Shape of the retinal surface in emmetropia and myopia," Invest Ophthalmol Vis Sci, 46(8), 2698-707 (2005).

[5] D. O. Mutti, R. I. Sholtz, N. E. Friedman et al., "Peripheral refraction and ocular shape in children," Invest Ophthalmol Vis Sci, 41(5), 1022-30 (2000).

[6] W. N. Charman, "Aberrations and myopia,” Ophthalmic Physiol Opt, 25(4), 285-301 (2005).

[7] W. N. Charman, and H. Radhakrishnan, "Peripheral refraction and the development of refractive error: a review," Ophthalmic Physiol Opt, 30(4), 321-38 (2010).

[8] D. O. Mutti, J. R. Hayes, G. L. Mitchell et al., "Refractive error, axial length, and relative peripheral refractive error before and after the onset of myopia," Invest Ophthalmol Vis Sci, 48(6), 2510-9 (2007).

[9] A. Seidemann, F. Schaeffel, A. Guirao et al., "Peripheral refractive errors in myopic, emmetropic, and hyperopic young subjects," J Opt Soc Am A Opt Image Sci Vis, 19(12), 2363-73 (2002).

[10] F. Schaeffel, and H. C. Howland, "Mathematical model of emmetropization in the chicken," J Opt Soc Am A, 5(12), 2080-6 (1988).

[11] E. L. Smith, 3rd, R. Ramamirtham, Y. Qiao-Grider et al., "Effects of foveal ablation on emmetropization and form-deprivation myopia," Invest Ophthalmol Vis Sci, 48(9), 3914-22 (2007).

[12] E. L. Smith, 3rd, L. F. Hung, and J. Huang, "Relative peripheral hyperopic defocus alters central refractive development in infant monkeys," Vision Res, 49(19), 2386-92 (2009).

[13] M. Bitzer, and F. Schaeffel, "Defocus-induced changes in ZENK expression in the chicken retina," Invest Ophthalmol Vis Sci, 43(1), 246-52 (2002).

[14] R. Schippert, and F. Schaeffel, "Peripheral defocus does not necessarily affect central refractive development," Vision research, 46(22), 3935-3940 (2006).

[15] D. O. Mutti, L. T. Sinnott, G. L. Mitchell et al., "Relative peripheral refractive error and the risk of onset and progression of myopia in children," Investigative ophthalmology \& visual science, 52(1), 199-205 (2011).

[16] C. C. Sng, X.-Y. Lin, G. Gazzard et al., "Peripheral refraction and refractive error in Singapore Chinese children," Investigative ophthalmology \& visual science, 52(2), 1181-1190 (2011).

[17] N. C. Strang, B. Cagnolati, and L. S. Gray, "Four year longitudinal investigation of ocular shape changes during refractive development," Investigative Ophthalmology \& Visual Science, 53(14), 139-139 (2012).

[18] J. C. Chen, B. Brown, and K. L. Schmid, "Evaluation of inner retinal function in myopia using oscillatory potentials of the multifocal electroretinogram," Vision research, 46(24), 4096-4103 (2006).

[19] N. J. Coletta, and T. Watson, "Effect of myopia on visual acuity measured with laser interference fringes," Vision Research, 46(5), 636-651 (2006).

[20] J. R. Heckenlively, and G. B. Arden, [Principles and practice of clinical electrophysiology of vision] MIT press, (2006).

[21] N. C. Strang, B. Winn, and A. Bradley, "The role of neural and optical factors in limiting visual resolution in myopia," Vision Research, 38(11), 1713-1721 (1998).

[22] H.-Y. Kuo, K. L. Schmid, and D. A. Atchison, "Visual backward masking performance in young adult emmetropes and myopes," Optometry \& Vision Science, 89(1), E90-E96 (2012).

[23] A. Mirzajani, E. Sarlaki, H. Kharazi et al., "Effect of lens-induced myopia on visual cortex activity: a functional MR imaging study," American Journal of Neuroradiology, 32(8), 1426-1429 (2011). 
[24] P. Bobak, I. Bodis-Wollner, and S. Guillory, "The effect of blur and contrast of VEP latency: comparison between check and sinusoidal grating patterns," Electroencephalography and Clinical Neurophysiology/Evoked Potentials Section, 68(4), 247-255 (1987).

[25] S. Sokol, and A. Moskowitz, "Effect of retinal blur on the peak latency of the pattern evoked potential," Vision research, 21(8), 1279-1286 (1981).

[26] L. Lundström, J. Gustafsson, I. Svensson et al., "Assessment of objective and subjective eccentric refraction," Optometry \& Vision Science, 82(4), 298-306 (2005).

[27] D. H. Brainard, "The psychophysics toolbox," Spatial vision, 10, 433-436 (1997).

[28] D. G. Pelli, "The VideoToolbox software for visual psychophysics: Transforming numbers into movies," Spatial vision, 10(4), 437-442 (1997).

[29] P. Wang, and D. Nikolic, "An LCD monitor with sufficiently precise timing for research in vision," Frontiers in human neuroscience, 5, 85 (2011).

[30] J. Shen, C. A. Clark, P. S. Soni et al., "Peripheral refraction with and without contact lens correction," Optometry and vision science: official publication of the American Academy of Optometry, 87(9), 642 (2010).

[31] L. Lundström, A. Mira-Agudelo, and P. Artal, "Peripheral optical errors and their change with accommodation differ between emmetropic and myopic eyes," Journal of Vision, 9(6), 17-17 (2009).

[32] L. N. Thibos, W. Wheeler, and D. Horner, "Power vectors: an application of Fourier analysis to the description and statistical analysis of refractive error," Optometry \& Vision Science, 74(6), 367-375 (1997).

[33] R. Fredericksen, P. J. Bex, and F. A. Verstraten, "How big is a Gabor patch, and why should we care?," JOSA A, 14(1), 1-12 (1997).

[34] A. B. Watson, and D. G. Pelli, "QUEST: A Bayesian adaptive psychometric method," Attention, Perception, \& Psychophysics, 33(2), 113-120 (1983).

[35] J. Rovamo, V. Virsu, P. Laurinen et al., "Resolution of gratings oriented along and across meridians in peripheral vision," Investigative Ophthalmology \& Visual Science, 23(5), 666-670 (1982).

[36] Q. Fan, Y.-Y. Teo, and S.-M. Saw, "Application of advanced statistics in ophthalmology," Investigative ophthalmology \& visual science, 52(9), 6059-6065 (2011).

[37] E. M. Harvey, V. Dobson, J. M. Miller et al., "Treatment of astigmatism-related amblyopia in 3-to 5-year-old children," Vision research, 44(14), 1623-1634 (2004).

[38] A. Whatham, F. Zimmermann, A. Martinez et al., "Influence of accommodation on off-axis refractive errors in myopic eyes," Journal of vision, 9(3), 14-14 (2009).

[39] R. F. Hess, and G. Maehara, "Does cognitive perception have access to brief temporal events?," i-Perception, 2(2), 142-149 (2011).

[40] J. P. Comerford, F. Thorn, and T. R. Corwin, "Effect of luminance level on contrast sensitivity in myopia," Optometry \& Vision Science, 64(11), 810-814 (1987).

[41] A. Ehsaei, C. M. Chisholm, I. E. Pacey et al., "Off axis partial coherence interferometry in myopes and emmetropes," Ophthalmic and Physiological Optics, 33(1), 26-34 (2013).

[42] N. S. Logan, B. Gilmartin, C. F. Wildsoet et al., "Posterior retinal contour in adult human anisomyopia," Investigative ophthalmology \& visual science, 45(7), 2152-2162 (2004).

[43] M. F. Silva, C. Mateus, A. Reis et al., "Asymmetry of visual sensory mechanisms: electrophysiological, structural, and psychophysical evidences," Journal of Vision, 10(6), 26-26 (2010).

[44] D. Allen, and R. F. Hess, "Is the visual field temporally homogeneous?," Vision research, 32(6), 1075-1084 (1992).

[45] D. Allen, R. F. Hess, and K. Nordby, "Is the rod visual field temporally homogeneous?," Vision research, 38(24), 3927-3931 (1998).

[46] P. Lewis, R. Rosén, P. Unsbo et al., "Resolution of static and dynamic stimuli in the peripheral visual field," Vision research, 51(16), 1829-1834 (2011).

[47] C. A. Curcio, and K. A. Allen, "Topography of ganglion cells in human retina," Journal of comparative Neurology, 300(1), 5-25 (1990).

[48] R. Calver, H. Radhakrishnan, E. Osuobeni et al., "Peripheral refraction for distance and near vision in emmetropes and myopes," Ophthalmic and Physiological Optics, 27(6), 584-593 (2007).

[49] P. Kang, Y. Fan, K. Oh et al., "Effect of single vision soft contact lenses on peripheral refraction," Optometry \& Vision Science, 89(7), 1014-1021 (2012).

[50] A. Mathur, D. A. Atchison, and W. N. Charman, "Effect of accommodation on peripheral ocular aberrations," Journal of Vision, 9(12), 20-20 (2009). 
[51] J. Gustafsson, and P. Unsbo, "Eccentric correction for off-axis vision in central visual field loss," Optometry \& Vision Science, 80(7), 535-541 (2003).

[52] R. S. Anderson, "The selective effect of optical defocus on detection and resolution acuity in peripheral vision," Current eye research, 15(3), 351-353 (1996).

[53] P. R. Lewis, K. Baskaran, R. Rosen et al., "The effect of refractive-correction on peripheral low-contrast resolution acuity," Investigative Ophthalmology \& Visual Science, 53(14), 4799-4799 (2012).

[54] E. M. Harvey, V. Dobson, C. E. Clifford-Donaldson et al., "Optical treatment of amblyopia in astigmatic children: the sensitive period for successful treatment," Ophthalmology, 114(12), 2293-2301 (2007).

[55] E. M. Harvey, "Development and treatment of astigmatism-related amblyopia," Optometry and vision science: official publication of the American Academy of Optometry, 86(6), 634 (2009).

[56] J. Jennings, and W. Charman, "The effects of central and peripheral refraction on critical fusion frequency," Ophthalmic and Physiological Optics, 1(2), 91-96 (1981).

[57] F. Faul, E. Erdfelder, A.-G. Lang et al., "G* Power 3: A flexible statistical power analysis program for the social, behavioral, and biomedical sciences," Behavior research methods, 39(2), 175-191 (2007). 


\section{SUPPLEMENTARY RESULTS}

Supplementary Table 1: Mean differences in dioptres of normalized J0 between positions-of-retina. Values on the table were obtained by subtracting $\mathrm{J} 0$ of position-of-retina specified in rows of the first column from J0 corresponding to the position-of-retina specified in the column heading.

\begin{tabular}{llllll}
\hline & $20 \mathrm{~T}$ & $10 \mathrm{~T}$ & $10 \mathrm{~N}$ & $20 \mathrm{~N}$ & $30 \mathrm{~N}$ \\
\hline $30 \mathrm{~T}$ & $-0.52 * * *$ & $-1.1 * * *$ & $-1.4^{* * *}$ & $-1.1^{* * *}$ & $-0.7^{* * *}$ \\
$20 \mathrm{~T}$ & & $-0.6^{* * *}$ & $-0.9 * * *$ & $-0.6^{* * *}$ & -0.1 \\
$10 \mathrm{~T}$ & & -0.3 & 0.0 & $0.4^{*}$ \\
$10 \mathrm{~N}$ & & & 0.3 & 0.7 \\
$20 \mathrm{~N}$ & & & & $0.5^{*}$ \\
\hline
\end{tabular}

*** The mean difference is significant at the 0.001 level; * The mean difference is significant at the 0.05 level

Supplementary Table 2: Mean differences in dioptres of normalized J45 between positions-of-retina. Values on the table were obtained by subtracting $\mathrm{J} 45$ of position-of-retina specified in rows of the first column from $\mathrm{J} 45$ corresponding to the position-of-retina specified in the column heading.

\begin{tabular}{llllll}
\hline & $20 \mathrm{~T}$ & $10 \mathrm{~T}$ & $10 \mathrm{~N}$ & $20 \mathrm{~N}$ & $30 \mathrm{~N}$ \\
\hline $30 \mathrm{~T}$ & 0.004 & 0.073 & $0.3^{* *}$ & $0.4^{* * *}$ & $0.5^{* * *}$ \\
$20 \mathrm{~T}$ & & 0.07 & $0.3^{* *}$ & $0.4^{* * *}$ & $0.5^{* * *}$ \\
$10 \mathrm{~T}$ & & $0.2^{*}$ & $0.4^{* * *}$ & $0.5^{* * *}$ \\
$10 \mathrm{~N}$ & & & 0.2 & $0.2^{* *}$ \\
$20 \mathrm{~N}$ & & & & 0.1 \\
\hline
\end{tabular}

*** The mean difference is significant at the 0.001 level; **The mean difference is significant at the 0.01 level; * The mean difference is significant at the 0.05 level 